Article

\title{
Passivity-Based Control Design for Magnetic Levitation System
}

\author{
Wang Yang ${ }^{(0)}$, Fanwei Meng *(D), Man Sun and Kai Liu ${ }^{\circledR}$ \\ School of Control Engineering, Northeastern University at Qinhuangdao, Qinhuangdao 066004, China; \\ yangwangzz@stumail.neu.edu.cn (W.Y.); sunman@neuq.edu.cn (M.S.); miaomiao@stumail.neu.edu.cn (K.L.) \\ * Correspondence: mengfanwei@neuq.edu.cn; Tel.: +86-173-3448-0916
}

Received: 2 March 2020; Accepted: 29 March 2020; Published: 1 April 2020

\begin{abstract}
The passivity-based control (PBC) is a new direction of nonlinear control, but the method is basically a qualitative method. A quantifiable design method in combination with PBC is provided in this paper. To solve the partial differential equation (PDE) for $\mathrm{PBC}$, the nonlinear system must first be transformed into a Hamiltonian model. The PDE for the Hamiltonian system is then quantifiably solved with an electromagnetic levitation example. The resulting control law is presented and discussed. The proposed method provides a practical design tool for nonlinear control.
\end{abstract}

Keywords: passivity-based control; interconnection and damping assignment; Hamiltonian system; nonlinear control; electromagnetic levitation system

\section{Introduction}

Due to many advantages of magnetic levitation system, including absence of contact, low noise, low energy consumption, it has been widely used, such as magnetic levitation bearings [1], magnetic levitation trains [2], magnetic levitation wind turbines [3], magnetic levitation vehicle operation lines [4]. Magnetic levitation system is highly nonlinear unstable system, it is a strong need to control the suspension gap. In recent years, various research methods have been proposed, such as Proportional-Integral-Derivative (PID) control [5,6], Linear Quadratic Regulator (LQR) control [7], and sliding mode controller [8], fuzzy control is presented in [9]. When PID controller is designed, the system has to be linearized, sliding mode control has chattering effect. As for fuzzy control, the membership function is selected entirely by experience.

In recent years, Ortega proposed a passivity-based design method for nonlinear system control based on the concept of energy variation in control systems [10,11]. The superiority of this design method has been recognized and gradually applied to various fields. Liu et al. [12,13] presented passivity-based controller to solve low-frequency voltage oscillation. Ortega et al. [14] applied sensorless passivity-based control to solve the trajectory tracking task associated with the bidirectional DC/DC Buck power converter-inverter-DC motor system. Zhang et al. [15] addressed the problem of stabilization of port-Hamiltonian systems via the ubiquitous PID controller. Jung et al. [16] designed a passivity-based robust switching controller for the posture stabilization of wheeled mobile robots with model uncertainty. Passivity-based theory and Lyapunov's stability theory can be used to study system stability. The passivity-based theory has unique advantages. For example, the storage function in passivity-based theory is related to the physical characteristics of the system, which is easy to obtain according to the structure of the system. Jung et al.'s method [16] is based on Lyapunov's method, has a complicated design process and needs to switch between two controllers. The passivity-based design idea is different from the traditional idea, it is based on (error) signal and (frequency) response. It retains more nonlinear characteristics. The physical meaning of the design process is clear, especially the structure of the system, Moreover, the structure of the controller is simple and easy to implement. 
Passivity-based control needs to solve a partial differential equation. In order to solve the problem easily, the mathematical model of the system needs to be transformed into the Hamilton model. However, passivity-based control is generally more focused on qualitative considerations and is more difficult to apply to specific designs. In this paper, passivity-based control is combined with the bias-based feedback control and the response characteristics of the system, combines qualitative and quantitative methods. The magnetic levitation system gap control (suspended at the expected value) is presented as an example to illustrate the detailed design process, and a practical design method for passivity-based control of nonlinear systems is provided.

The rest of paper is organized as follows. Section 2 briefly introduces passivity-based control. In Section 3, we briefly introduce sensitivity function. Passivity-based control for magnetic levitation systems is presented in Section 4. In Section 5, we analysis the role of the interconnection and damping assignment. The concluding remarks are contained in Section 6.

\section{Passivity-Based Control}

If there is a non-negative storage function $H$ that satisfies the following equation, the system is said to be passive.

$$
H[x(t)]-H[x(0)] \leq \int_{0}^{t} \boldsymbol{u}^{T}(s) \boldsymbol{y}(s) d s
$$

where $\boldsymbol{u} \in \mathbb{R}^{m}$ is the control input of the system, $y \in \mathbb{R}^{m}$ is the output of the system.

Equation (1) can also be written as an energy balance equation

$$
H[\boldsymbol{x}(t)]-H[\boldsymbol{x}(0)]=\int_{0}^{t} \boldsymbol{u}^{T}(s) \boldsymbol{y}(s) d s-d(t)
$$

where the left term of Equation (2) represents the energy stored by the system, the integral term of the right term represents the energy supplied, and $d(t)$ is the energy dissipated. $H_{a}(x)$

If select a control action $\boldsymbol{u}=\boldsymbol{\beta}(\boldsymbol{x})+\boldsymbol{v}$, and let $\boldsymbol{\beta}(\boldsymbol{x})$ satisfies the following equation about function

$$
-\int_{0}^{t} \boldsymbol{\beta}^{T}[\boldsymbol{x}(s)] \boldsymbol{y}(s) d s=H_{a}[\boldsymbol{x}(t)]+n_{0}
$$

where $n_{0}$ is the integral constant. Substituting the above $u$ into Equation (2), we have that

$$
H_{d}[\boldsymbol{x}(t)]-H_{d}[\boldsymbol{x}(0)]=\int_{0}^{t} \boldsymbol{v}^{T}(s) \boldsymbol{y}(s) d s-d(t)
$$

where Equation (4) shows that under this control, the new input $v$ to output $y$ mapping is still passive. Then, the new energy function is written as

$$
H_{d}(\boldsymbol{x}) \triangleq H(\boldsymbol{x})+H_{a}(\boldsymbol{x})
$$

If Equation (5) has a minimum value at the desired working point $x_{0}$, then when $\boldsymbol{v} \equiv \boldsymbol{0}, x_{0}$ is a stable equilibrium point, and $H_{d}(\boldsymbol{x})$ is the Lyapunov function. Note that $H_{d}(\boldsymbol{x})$ is configurable as required, and the dissipative term $d(t)$ can also be reconfigured. This is the basic idea of passivity-based control (PBC).

In general, Equation (3) needs to solve a partial differential equation (PDE). But for a general nonlinear passive system $\sum_{1}$,

$$
\sum_{1}:\left\{\begin{array}{l}
\dot{x}=f(x)+g(x) u \\
y=h(x)
\end{array}\right.
$$


This PDE is not easy to solve, so under the premise of the above PBC design, Equation (6) of the system need to be organized into a port-controllable Hamilton system, $\Sigma_{2}$ [10],

$$
\sum_{2}:\left\{\begin{array}{l}
\dot{x}=[J(x)-R(x)] \frac{\partial H}{\partial x}(x)+g(x) u \\
y=g^{T}(x) \frac{\partial H}{\partial x}(x)
\end{array}\right.
$$

where $H(x)$ is the energy function of the system, $\boldsymbol{J}(\boldsymbol{x})=-\boldsymbol{J}^{T}(\boldsymbol{x})$ represents the interconnection structure of the system, and $\boldsymbol{R}(\boldsymbol{x})=\boldsymbol{R}^{T}(\boldsymbol{x}) \geq 0$ is the dissipative matrix, which represents the dissipative structure of the system, damping in English, which means that the energy is dissipated and quickly stabilized.

For the Hamilton system, its partial differential equations are easier to solve, because $\boldsymbol{J}$ and $\boldsymbol{R}$ can reflect the structural characteristics of the system and facilitate PBC design. Such a design is called interconnection and damping assignment $\mathrm{PBC}$, abbreviated as IDA-PBC.

Let the Hamilton system $\Sigma_{2}$ [Equation (7)] select static feedback $\boldsymbol{u}=\boldsymbol{\beta}(\boldsymbol{x})$, then the equation for the state $x$ of the system can be written as

$$
\dot{x}=[J(x)-R(x)] \frac{\partial H}{\partial x}(x)+g(x) \beta(x)
$$

The following partial differential equation what is required now is

$$
[\boldsymbol{J}(\boldsymbol{x})-\boldsymbol{R}(\boldsymbol{x})] \frac{\partial H_{a}}{\partial x}(\boldsymbol{x})=\boldsymbol{g}(\boldsymbol{x}) \boldsymbol{\beta}(\boldsymbol{x})
$$

If $\beta(x)$ can be solved from Equation (9), and according to Equation (5), then Equation (8) can be rewritten as

$$
\dot{x}=[\boldsymbol{J}(x)-R(x)] \frac{\partial H_{d}}{\partial x}(x)
$$

is that there is a new energy function, $H_{d}(\boldsymbol{x})=H(\boldsymbol{x})+H_{a}(\boldsymbol{x})$. If it is guaranteed that $H_{d}(\boldsymbol{x})$ can be minimized at the equilibrium point, then this state feedback $\boldsymbol{u}=\boldsymbol{\beta}(\boldsymbol{x})$ will stabilize the system at that equilibrium point.

In fact, $\boldsymbol{J}(\boldsymbol{x})$ and $\boldsymbol{R}(\boldsymbol{x})$ in Equation (10) do not have to remain unchanged, and the dynamic characteristics of the closed-loop system can be changed as required

$$
\dot{x}=\left[J_{d}(x)-R_{d}(x)\right] \frac{\partial H_{d}}{\partial x}(x)
$$

is that the Hamilton system's interconnect relationship $J_{d}(x)=-J_{d}^{T}(x)$ and the dissipative term $\boldsymbol{R}_{\boldsymbol{d}}(\boldsymbol{x})=\boldsymbol{R}_{\boldsymbol{d}}^{T}(\boldsymbol{x}) \geq 0$ can be reconfigured. This is IDA-PBC. IDA-PBC not only increases the freedom of design, but also makes the solution of partial differential equations more flexible due to the addition of some new parameters.

According to Equations (8) and (11), the partial differential equation in this IDA-PBC problem now is

$$
\left[J_{d}(x)-R_{d}(x)\right] \frac{\partial H_{a}}{\partial x}(x)=-\left[J_{a}(x)-R_{a}(x)\right] \frac{\partial H}{\partial x}(x)+g(x) \boldsymbol{\beta}(x)
$$

where

$$
J_{a}(x) \triangleq J_{d}(x)-J(x), R_{a}(x) \triangleq R_{d}(x)-R(x)
$$

The partial differential Equation (12) is generally not easy to solve directly. In order to solve $\partial H_{a} / \partial x$, the literature [10] proposes some additional conditions such as integrable, equilibrium point conditions and Lyapunov stability conditions. But such a solution often causes the solution to lose its physical meaning. In fact, for some less complicated occasions, Equation (12) can be solved directly. The directly obtained solution has a clear physical concept and is convenient for IDA-PBC design, making it easier to determine specific parameters. The following is a detailed description in conjunction with a design example. 


\section{Performance: Sensitivity Function}

The feedback control system block diagram is shown in Figure 1, where $r$ is the reference input, $e$ is the error signal, $K$ is a controller, $u$ is control law, $G$ is the plant requiring control, $y$ is the system output.

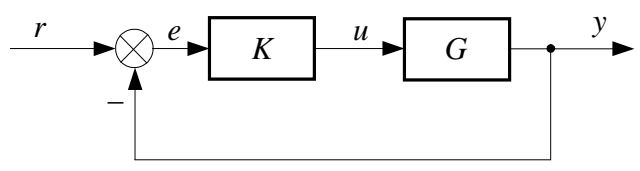

Figure 1. Feedback control system.

The sensitivity function $S$ of the system is defined as:

$$
S=\frac{\mathrm{d} \ln T}{\mathrm{~d} \ln G}=\frac{\mathrm{d} T / T}{\mathrm{~d} G / G}=\frac{G}{T} \frac{\mathrm{d} T}{\mathrm{~d} G}=\frac{1}{1+K G}
$$

where $T$ is the closed-loop transfer function of the system. We can know that the system sensitivity quantitatively represents the degree of sensitivity of the closed-loop transfer function $T$ relative to the plant parameter changes. If the system sensitivity is low, the design is robust to plant modeling errors. If $S$ is seen as the transfer function, it is characterized by the capability of the system to track the input signal (the transfer function from the reference input $r$ to the error signal $e$ is equal to the sensitivity) and the disturbance rejection ability (if there is a disturbance at the output, the transfer function from the disturbance to the system output $y$ is also equal to the sensitivity). It is thought that the sensitivity should be as small as possible. The design should be such that the system achieves the goal of better feedback features, specifically, low sensitivity and good disturbance rejection ability. In addition to the description above, the sensitivity function also reflects the other important features of the system, and it is a very important design indicator. When an actual system is designed, it is necessary to reasonably design the peak value of the sensitivity function and the frequency of the peak value. However, the sensitivity function cannot be arbitrarily specified in the design. In the comprehensive design of a system, one important problem often encountered is the degree to which the system performance can be achieved [17]. The gap of the magnetic levitation system is generally small. If the anti-interference ability or the robustness is poor, collisions are likely to occur and the system cannot run stably. If it is in a magnetic levitation train, then a serious accident will occur. Reduced sensitivity is necessary. The optimal sensitivity function characteristics are given in the paper. In Section 4, in order to obtain a better sensitivity function, $c_{1}, \alpha, R_{\alpha}$ is reasonably selected. $\alpha$ (in Equation (30)) provides the coupling of displacement and velocity to the flux linkage, making system (29) a passive system, $R_{\alpha}$ is added to the dissipative matrix (31) to increase the dissipation of energy and improve the response speed, negative feedback $-c_{1} \widetilde{x}_{1}(39,40)$ is applied to the flux linkage that forms the electromagnetic force to stabilize.

\section{Passivity-Based Control for Magnetic Levitation System}

Magnetic levitation system are often used to verify control algorithms [18-20]. The simplified magnetic levitation control system model is shown in Figure 2 [21], $m$ is the mass of the suspended magnet, $i$ is the current of the electromagnet coil, $u$ is the input voltage, $f$ is the electromagnetic levitation force, $z$ is the distance between the electromagnet and the track, $N$ is the number of coil windings, $R_{0}$ is the coil resistance, $A$ is the effective area of the magnetic pole, $R_{T}$ is the air gap resistance, and $\Phi_{T}$ is the main pole flux. 


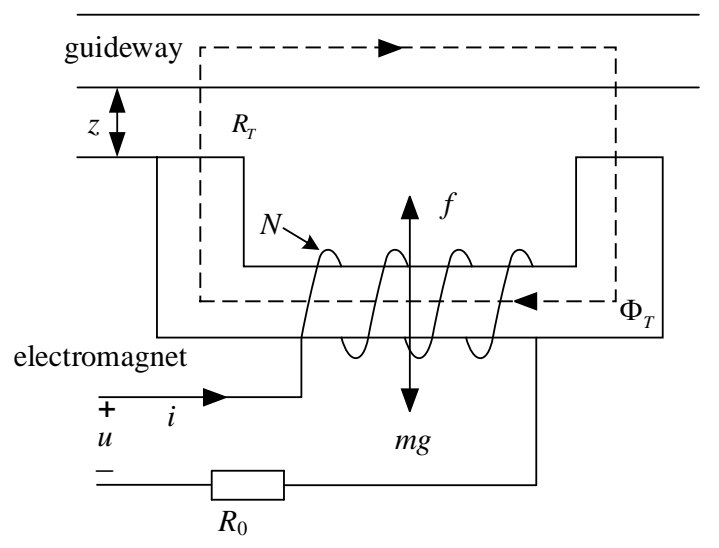

Figure 2. Model of the electromagnetic levitation system.

Neglecting the leakage flux and the magnetic reluctance of the guide rail and the magnetic core, the magnetic resistance of the magnetic circuit formed by the electromagnet and the guide rail is mainly concentrated on the air gap between the two, so the effective air gap resistance $R_{T}$ is written as

$$
R_{T}=\frac{2 z}{\mu_{0} A}
$$

where $\mu_{0}$ is the permeability of vacuum, and $\mu_{0}=4 \pi \times 10^{-7} \mathrm{H} / \mathrm{m}$. The flux linkage of electromagnet is written as

$$
\lambda=N \Phi_{T}=L i
$$

According to Equations (13) and (14), the inductance of the electromagnet winding satisfies

$$
L(z)=\frac{N \Phi_{T}}{i}=\frac{N}{i} \times \frac{N i}{R_{T}}=\frac{\mu_{0} N^{2} A}{2 z}
$$

According to Equation (15), the energy storage of the magnetic field is as follows

$$
E(z, i)=\frac{1}{2} L(z) i^{2}=\frac{1}{2} \frac{\mu_{0} N^{2} A}{2 z} i^{2}
$$

Therefore, the electromagnetic force can be calculated from Equation (16) as follows (the derivative of energy versus displacement $z$ )

$$
f(z, i)=-\frac{\partial E}{\partial z}=\frac{\mu_{0} N^{2} A}{4}\left[\frac{i}{z}\right]^{2}
$$

Assuming that the degree of freedom of the dissipative system is $n$, the general form of the Lagrange equation is

$$
\frac{\mathrm{d}}{\mathrm{d} t}\left(\frac{\partial L}{\partial \dot{r}_{j}}\right)-\frac{\partial L}{\partial r_{j}}=-\frac{\partial G}{\partial \dot{r}_{j}}+Q_{j} j=1, \ldots \ldots, n
$$

where $j$ is the label of the generalized coordinate, $L$ is the Lagrange function, $r$ and $\dot{r}$ are the generalized coordinate and the generalized velocity, respectively, $G$ is the dissipative function of the system, $Q_{j}$ is a generalized and nonpotential force (except the dissipative force).

In the magnetic levitation system, let $r_{1}=z$ and $r_{2}=q$ are generalized coordinates, and $\dot{s}$ and $\dot{q}=i$ are the corresponding generalized velocities.

The kinetic energy of the system can be written as

$$
T=\frac{1}{2} m \dot{z}^{2}+E(z, i)
$$


The potential energy can be written as

$$
V=-m g z
$$

Using Equations (19) and (20), we can formulate Lagrange function of the system as

$$
L=T-V=\frac{1}{2} m \dot{z}^{2}+E(z, i)+m g z
$$

The system's dissipation function is given by

$$
G=\frac{1}{2} R_{0} i^{2}
$$

For generalized coordinates $r_{1}$ and $r_{2}$, the following equations hold

$$
Q_{1}=0, Q_{2}=u
$$

Substituting Equations (21)-(23) into Equation (18), nonlinear model of the system will be

$$
\begin{gathered}
\ddot{z}=-\frac{k i^{2}}{4 m z^{2}}+g \\
\dot{i}=\frac{2 z}{k}\left(-R_{0} i+u\right)+\frac{i}{z} \dot{z}
\end{gathered}
$$

According to Equations (14), (15), (24), and (25), we obtain Equation (26)

$$
\sum_{3}:\left\{\begin{array}{l}
\ddot{z}=-\frac{\lambda^{2}}{k m}+g \\
\dot{\lambda}=-\frac{2 R_{0}}{k} \lambda z+u
\end{array}\right.
$$

where $\lambda$ is the flux linkage of electromagnet, $k$ is a constant, and $k=\mu_{0} A N^{2}=0.0010 \mathrm{H} \cdot \mathrm{m}$. The nominal working point of the system is $z_{0}=4 \mathrm{~mm}, i_{0}=3.0538 \mathrm{~A}$ and $\lambda_{0}=0.3851 \mathrm{~V} \cdot \mathrm{sec}$.

Then the inductance of the system is $L(z)=k / 2 z$, the magnetic energy is $\lambda^{2} / 2 L(z)=\lambda^{2} z / k$, so the total energy $H(\lambda, z, \dot{z})$ of the system can be written as

$$
H(\lambda, z, \dot{z})=\frac{\lambda^{2} z}{k}-m g z+\frac{1}{2} m \dot{z}^{2}
$$

where the second term in the formula is potential energy, in this example, the positive direction of $z$ points downward. The third term in the formula is kinetic energy. Taking the state variable $x=(\lambda, z, \dot{z})^{T}$, the energy function can be written as

$$
H(x)=\frac{x_{1}^{2} x_{2}}{k}-m g x_{2}+\frac{1}{2} m x_{3}^{2}
$$

The partial derivative of Equation (27) is as follows

$$
\frac{\partial H}{\partial x}(x)=\left[\frac{2 x_{1} x_{2}}{k}, \frac{x_{1}^{2}}{k}-m g, m x_{3}\right]^{T}
$$

According to Equation (28), Equation (26) can be organized into a port-controllable Hamilton model, as shown in Equation (7) 


$$
\begin{aligned}
\dot{x} & =[\boldsymbol{J}(\boldsymbol{x})-\boldsymbol{R}(\boldsymbol{x})] \frac{\partial H}{\partial x}(\boldsymbol{x})+\boldsymbol{g}(\boldsymbol{x}) \boldsymbol{u} \\
& =\left(\left(\begin{array}{ccc}
0 & 0 & 0 \\
0 & 0 & 1 / m \\
0 & -1 / m & 0
\end{array}\right)-\left(\begin{array}{ccc}
R_{0} & 0 & 0 \\
0 & 0 & 0 \\
0 & 0 & 0
\end{array}\right)\right) \frac{\partial H}{\partial x}(\boldsymbol{x})+\left(\begin{array}{l}
1 \\
0 \\
0
\end{array}\right) u
\end{aligned}
$$

Note that the original system Equation (29) is not a passive system, so the interconnection matrix needs to be configured into

$$
\boldsymbol{J}_{\boldsymbol{d}}(\boldsymbol{x})=\boldsymbol{J}(\boldsymbol{x})+\boldsymbol{J}_{\boldsymbol{a}}(\boldsymbol{x})=\left(\begin{array}{ccc}
0 & 0 & \alpha \\
0 & 0 & 1 / m \\
-\alpha & -1 / m & 0
\end{array}\right)
$$

where $\alpha$ provides the coupling of displacement and velocity to the flux linkage, making it a passive system, as discussed in Figure 3 below. In addition, it is also necessary to add $R_{\alpha}$ to the dissipative matrix to increase the dissipation of energy and improve the response speed, that is

$$
\boldsymbol{R}_{\boldsymbol{d}}(\boldsymbol{x})=\boldsymbol{R}(\boldsymbol{x})+\boldsymbol{R}_{\boldsymbol{a}}(\boldsymbol{x})=\left(\begin{array}{ccc}
R_{0} & 0 & 0 \\
0 & R_{\alpha} & 0 \\
0 & 0 & 0
\end{array}\right)
$$

Substituting Equations (30) and (31) into Equation (12) to obtain the partial differential equation of this example is

$$
\begin{aligned}
& \left(\begin{array}{ccc}
-R_{0} & 0 & \alpha \\
0 & -R_{\alpha} & 1 / m \\
-\alpha & -1 / m & 0
\end{array}\right) \frac{\partial H_{a}}{\partial x}(x) \\
& =-\left(\begin{array}{ccc}
0 & 0 & \alpha \\
0 & -R_{\alpha} & 0 \\
-\alpha & 0 & 0
\end{array}\right)\left(\begin{array}{c}
\frac{2 x_{1} x_{2}}{k} \\
\frac{x_{1}^{2}}{k}-m g \\
m x_{3}
\end{array}\right)+\left(\begin{array}{l}
1 \\
0 \\
0
\end{array}\right) \beta(x)
\end{aligned}
$$

After Equation (32) is expanded, we obtain

$$
\begin{gathered}
-R_{0} \frac{\partial H_{a}}{\partial x_{1}}+\alpha \frac{\partial H_{a}}{\partial x_{3}}=-\alpha m x_{3}+\beta(x) \\
-R_{\alpha} \frac{\partial H_{a}}{\partial x_{2}}+\frac{1}{m} \frac{\partial H_{a}}{\partial x_{3}}=R_{\alpha}\left(\frac{x_{1}^{2}}{k}-m g\right) \\
-\alpha \frac{\partial H_{a}}{\partial x_{1}}-\frac{1}{m} \frac{\partial H_{a}}{\partial x_{2}}=\frac{2 \alpha}{k} x_{1} x_{2}
\end{gathered}
$$

where Equation (33) can be used to determine the control action $u=\beta(x)$, Equation (34) and (35) are the partial differential equations that need to be solved. Note that the energy function $H_{d}(x)$ of the final closed-loop system is a Lyapunov function and there should be no totals for $x_{1}$ (flux linkage) and $x_{2}$ (distance), so according to Equation (5), we should use $H_{a}$ to subtract these two terms of $H(\boldsymbol{x})$ [Equation (27)]. According to Equations (34) and (35), it can be known that $H_{a}(x)$ should also have the following function term $\Phi$, and use the equality of the left and right terms to solve this system of equations. In this way, the final solution is written as

$$
H_{a}(x)=-\frac{x_{1}^{2} x_{2}}{k}+m g x_{2}-\frac{g}{\alpha} x_{1}+\frac{x_{1}^{3}}{3 \alpha m k}+\Phi\left(-\frac{1}{\alpha} x_{1}+m x_{2}+m^{2} R_{\alpha} x_{3}\right)
$$


where the function $\Phi$ in Equation (36) should have the synthesized function $H_{d}(x)$, a minimum at the equilibrium point $\left(x_{10}, x_{20}, 0\right)$, so that $H_{d}(\boldsymbol{x})$ becomes the Lyapunov function, so let $\Phi$ be a square form

$$
\begin{aligned}
& \Phi\left(-\frac{1}{\alpha} x_{1}+m x_{2}+m^{2} R_{\alpha} x_{3}\right) \\
& =q\left(-\frac{1}{\alpha}\left(x_{1}-x_{10}\right)+m\left(x_{2}-x_{20}\right)+m^{2} R_{\alpha} x_{3}\right)^{2} \\
& =q\left(-\frac{1}{\alpha} \widetilde{x}_{1}+m \widetilde{x}_{2}+m^{2} R_{\alpha} x_{3}\right)^{2}
\end{aligned}
$$

where $q$ is a constant, the tilde indicates increment, $\widetilde{x}_{1}=x_{1}-x_{10}, \widetilde{x}_{2}=x_{2}-x_{20}$.

After adding feedback, the new energy function of the closed-loop system is

$$
H_{d}(\boldsymbol{x})=H(\boldsymbol{x})+H_{a}(\boldsymbol{x})=\frac{1}{2} m x_{3}^{2}+\frac{x_{1}^{3}}{3 \alpha m k}-\frac{g}{\alpha} x_{1}+q\left(-\frac{1}{\alpha} \widetilde{x}_{1}+m \widetilde{x}_{2}+m^{2} R_{\alpha} x_{3}\right)^{2}
$$

Substituting the function $\Phi$ of Equation (37) into Equation (36) of $H_{a}(\boldsymbol{x})$, and then in accordance with Equation (33), the control action can be obtained

$$
\begin{aligned}
u & =\beta(x) \\
& =-R_{0} \frac{\partial H_{a}}{\partial x_{1}}+\alpha \frac{\partial H_{a}}{\partial x_{3}}+\alpha m x_{3} \\
& =R_{0}\left(\frac{2 x_{1} x_{2}}{k}\right)+\frac{R_{0}}{\alpha}\left(g-\frac{x_{1}^{2}}{m k}\right)-c_{1} \widetilde{x}_{1}+\alpha m c_{1} \widetilde{x}_{2}+\alpha m\left(1+m R_{\alpha} c_{1}\right) x_{3}
\end{aligned}
$$

where

$$
c_{1}=q\left(\frac{2 R_{0}}{\alpha^{2}}+2 m^{2} R_{\alpha}\right)
$$

This control action $u$ can be explained as follows. The first term of Equation (39) is equal to $R_{0} i$, which is used to compensate for the voltage drop across resistor $R_{0}$, the second term is the acceleration $\ddot{z}$ [see Equation (26)]. Therefore, it can be seen from Equation (39) that the control action after compensating for the voltage drop is equivalent to the conventional deviation control with respect to the equilibrium point $\left(x_{10}, x_{20}, 0\right)$. Specifically, first, negative feedback $-c_{1} \widetilde{x}_{1}$ is first applied to the flux linkage that forms the electromagnetic force to stabilize, and the rest are displacement $\left(\widetilde{x}_{2}\right)$, velocity $\left(x_{3}\right)$, and acceleration $(\ddot{z})$. It can be seen that the design results for this passivity can be explained. The difference is that this passivity-based design provides a new, clear physical concept (referred to as energy concept) design idea for the control of nonlinear systems, as further explained below.

In this example, the first term of the control action Equation (39) compensates for the nonlinear term in the flux linkage loop, so the nonlinear term in the equation $\sum_{3}$ of the system leaves only the square of the electromagnetic force generated by the flux linkage $\lambda$

$$
\ddot{z}=-\frac{\lambda^{2}}{k m}+g
$$

Equation (41) can be linearized, so the parameter relationship in the control action is first analyzed from the linearization system. Finally, the configuration of the inline structure $J_{d}$ and the dissipative matrix $R_{d}$ in IDA-PBC is discussed based on this relationship.

Linearizing $f=\lambda^{2} / k$ in Equation (41), take one degree term

$$
\left.\frac{\partial f}{\partial \lambda}\right|_{0} \Delta \lambda=\frac{2 \lambda_{0}}{k} \Delta \lambda=763.45 \Delta \lambda
$$

After the voltage drop is compensated, and the control action Equation (39) is added, according to Equation (42), the signal flow diagram under the small deviation linearization of the system as shown in Figure 3. 


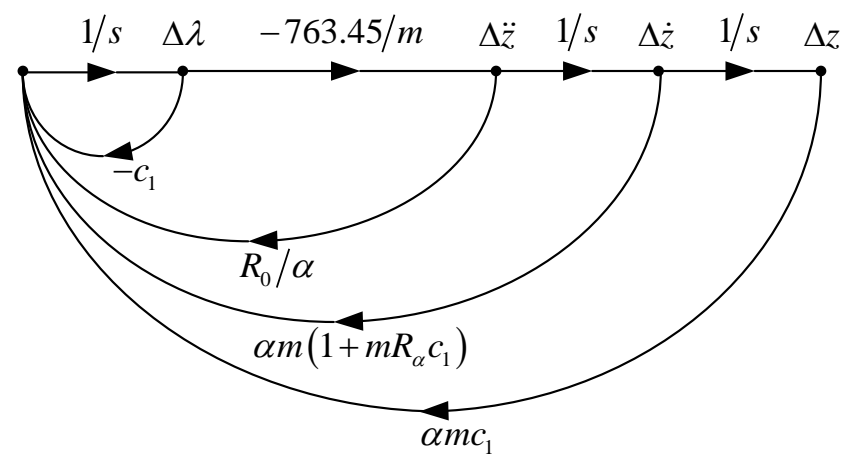

Figure 3. Signal-flow diagram of the (PBC) system.

For the sake of discussion, the parameters in the system are specifically taken as $c_{1}=200, \alpha=16$, $R_{\alpha}=0.0005$. Under this set of parameters, the acceleration loop of $R_{0} / \alpha$ has less influence in the system analysis, so it is omitted. Thus, the open loop characteristic of the system corresponding to Figure 3 is written as

$$
G(s)=\frac{763.45 \alpha c_{1}}{s\left[s\left(s+c_{1}\right)+763.45 \alpha\left(1+m R_{\alpha} c_{1}\right)\right]}
$$

We obtain the natural frequency of the inner loop (rate loop) obtained by Equation (43)

$$
\omega_{i}=\sqrt{763.45 \alpha\left(1+m R_{\alpha} c_{1}\right)}=175 \mathrm{rad} / \mathrm{sec}
$$

The low frequency band approximation of $G(s)$ is

$$
G(s) \approx \frac{c_{1}}{\left(1+m R_{\alpha} c_{1}\right) s}=\frac{80}{s}
$$

is that the system crosses the frequency of the $0 \mathrm{~dB}$ line. Figure 4 shows the Bode diagram of this example.

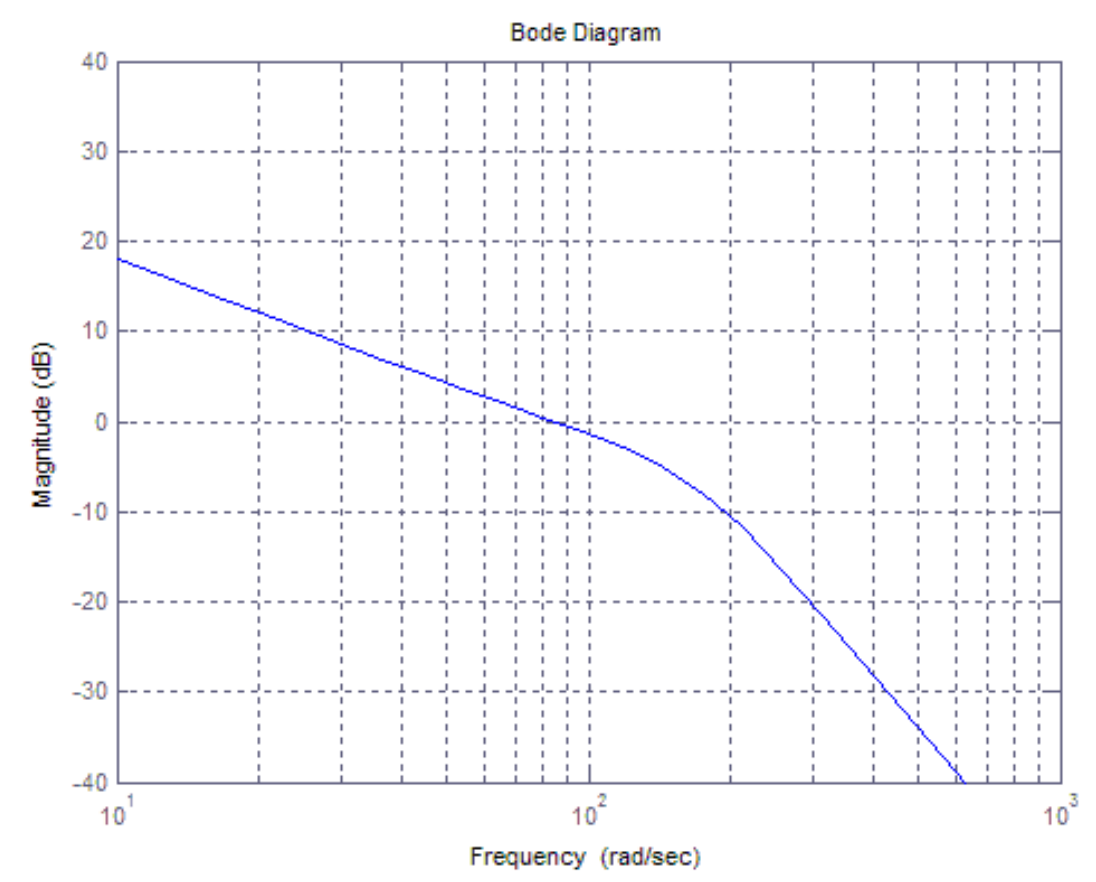

Figure 4. Bode plot of the system. 


\section{Discussion}

From the signal flow diagram and the above data, it can be seen that the roles of the interconnection and damping assignment is:

(1) $\alpha$ exists in the feedback gains of $z$ and $\dot{z}$, indicating that $\alpha$ is configured in $J_{d}(\boldsymbol{x})$ to provide channels for $z$ and $\dot{z}$. With these two channels, the entire system is likely to become a passive system.

(2) The feedback gains of $\lambda, z$ and $\dot{z}$ are all related to $c_{1}$, so the value of $c_{1}$ directly determines the response speed of the system. This can be seen Equation (40), $c_{1}$ contains the dissipation coefficients $R_{0}$ and $R_{\alpha}$, especially $R_{\alpha}$ is added to the dissipative matrix $R_{d}(x)$. The role of the dissipative matrix is to accelerate the energy dissipation, so that make it quickly converge to the equilibrium point.

(3) It can be seen from Equation (38) that the relative relationship between the variables in the quadratic term is consistent with the relative relationship between the feedback gains of $\lambda, z, \dot{z}$ in Figure 3, indicating that the gain of the feedback loop is closely related to the composition of the Lyapunov function $H_{d}(\boldsymbol{x})$.

The performance of the IDA-PBC system can be seen from this example that the nonlinear term in $H_{a}(x)$ [Equation (36)] is mainly used to cancel a part of the nonlinear characteristics of the object, and the other function $\Phi$ is a quadratic form. Therefore, $u=\beta(x)$ obtained by using Equation (39) and the increments are linear. Therefore, although it is a nonlinear object, due to the linear feedback, the nonlinear influence of the forward link is weakened by the feedback, so that the whole system exhibits approximately linear characteristics. Figure 5 is a graph showing the sensitivity characteristics of the output of the system in this example. This set of curves is obtained by simulation of the actual nonlinear model. It is based on the curve of the ratio of the sinusoidal output to the sinusoidal input at different frequencies. The amplitude of the signal during the test was $0.1 \mathrm{~mm}, 0.5 \mathrm{~mm}, 0.8 \mathrm{~mm}$, and $1 \mathrm{~mm}$. As the amplitude of the signal increases, the sensitivity $|S(j \omega)|$ peak shifts slightly to the left. This is because the squared term causes the gains in the positive and negative directions to be different, resulting in a decrease in the equivalent gain. In general, however, the sensitivity characteristics at different amplitudes does not change significantly. The time domain response of the system is shown in Figure 6.

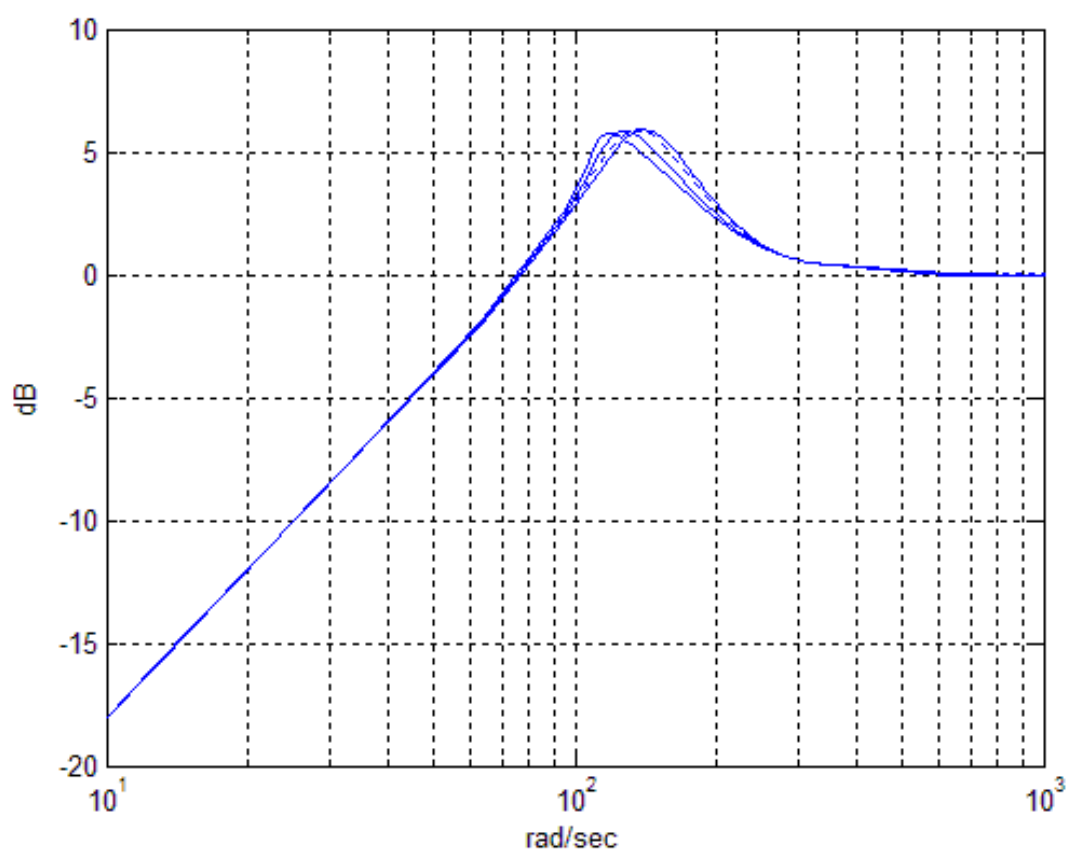

Figure 5. Sensitivity plots of the real nonlinear system. 


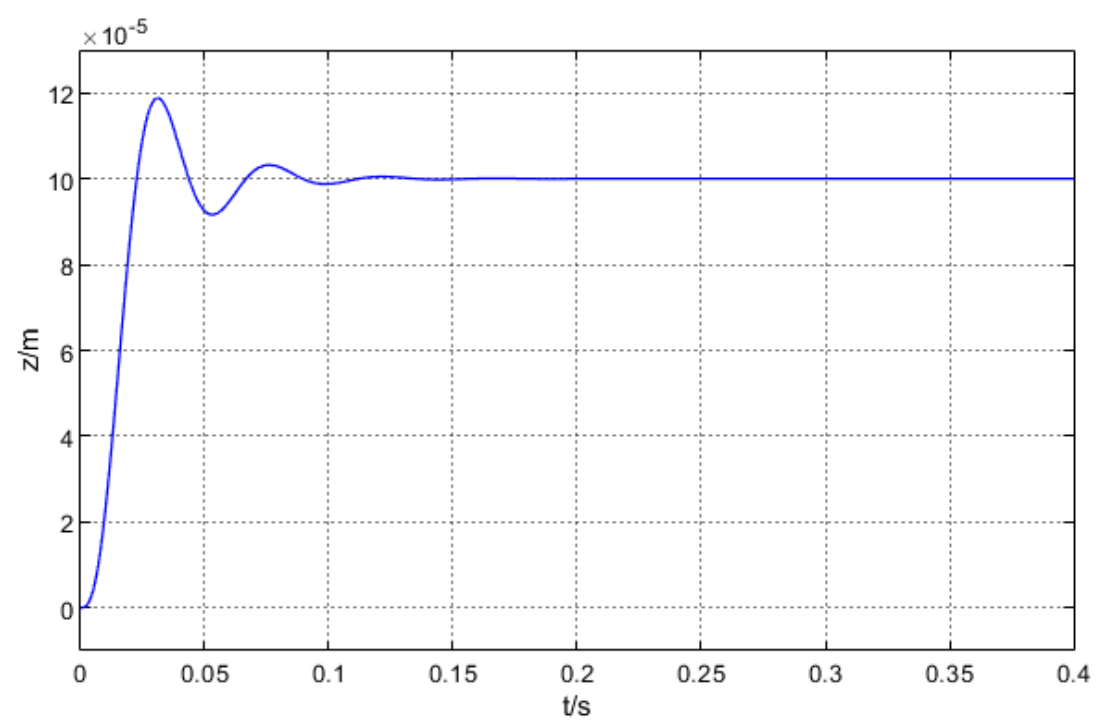

Figure 6. Time domain response of the system.

\section{Conclusions}

(1) In this paper, the passivity-based control of magnetic levitation system clearly reveals the relationship between the internal matrix and the dissipative matrix and the performance of the system, IDA-PBC is biased towards structural, or qualitative considerations of system design. If IDA-PBC is combined with the conventional idea of (error) signal response (see Figures 3 and 4), it can be an effective method for control design of nonlinear systems. It also reveals the connection between the construction of the Lyapunov function and the feedback control. It also provides a basis for quantitative design for passivity-based control.

(2) The control action derived from passivity-based control is basically linear, so the nonlinear system uses passivity-based control to obtain the response characteristics of an approximate linear system.

Author Contributions: Conceptualization, W.Y. and F.M.; methodology, W.Y. and F.M.; software, F.M.; validation, M.S. and K.L.; writing—original draft preparation, F.M.; writing—review and editing, F.M.; visualization, M.S. and K.L.; supervision, F.M. and M.S. All authors have read and agreed to the published version of the manuscript.

Funding: This research was funded by the Fundamental Research Funds for the Central Universities (No. N182304010) and the Natural Science Foundation of Hebei Province (No. F2019501012).

Conflicts of Interest: The authors declare no conflict of interest.

\section{References}

1. Martins, F.G.R.; Sass, F.; Ferreira, A.C.; de Andrade, R. A novel magnetic bearing using REBCO double crossed loop coils. IEEE Trans. Appl. Supercond. 2018, 28, 1-5. [CrossRef]

2. Zhai, M.; Long, Z.; Li, X. A New Strategy for Improving the Tracking Performance of Magnetic Levitation System in Maglev Train. Symmetry 2019, 11, 1053. [CrossRef]

3. Ahmad, G.; Amin, U. Design, construction and study of small scale vertical axis wind turbine based on a magnetically levitated axial flux permanent magnet generator. Renew. Energy 2017, 101, 286-292. [CrossRef]

4. Sotelo, G.G.; de Oliveira, R.A.H.; Costa, F.S.; Dias, D.H.N.; de Andrade, R.; Stephan, R.M. A Full Scale Superconducting Magnetic Levitation (MagLev) Vehicle Operational Line. IEEE Trans. Appl. Supercond. 2015, 25, 1-5. [CrossRef]

5. Bhaduri, R.; Banerjee, S.; Sarkar, M.K. Genetic Algorithm Based Optimization of Controller Parameters for an Electromagnetic Levitation System. In Proceedings of the Advanced Materials Research; Trans Tech Publishers: Zurich, Switzerland, 2012; Volume 403, pp. 3900-3908.

6. Aghazadeh, A.; Niazazari, I.; Askarian Abyaneh, H. Tuned Parameters of PID for Optimization of Losses in Magnetic Levitation System. Int. J. Railw. Res. 2019, 6, 29-37. 
7. Kumare, V.; Jerome, J. Algebraic Riccati equation based Q and R matrices selection algorithm for optimal LQR applied to tracking control of 3rd order magnetic levitation system. Arch. Electr. Eng. 2016, 65, 151-169. [CrossRef]

8. Sathiyavathi, S. Design of sliding mode controller for magnetic levitation system. Comput. Electr. Eng. 2019, 78, 184-203.

9. Bojan-Dragos, C.-A.; Precup, R.-E.; Hergane, S.; Teban, T.-A.; Petriu, E.M. Fuzzy logic-based adaptive control scheme for magnetic levitation systems. In Proceedings of the 2017 IEEE International Symposium on Robotics and Intelligent Sensors (IRIS), Ottawa, ON, Canada, 5-7 October 2017; pp. 160-165.

10. Ortega, R.; Van Der Schaft, A.J.; Mareels, I.; Maschke, B. Putting energy back in control. IEEE Control Syst. Mag. 2001, 21, 18-33.

11. Ortega, R.; van der Schaft, A.; Maschke, B.; Escobar, G. Interconnection and damping assignment passivity-based control of port-controlled Hamiltonian systems. Automatica 2002, 38, 585-596. [CrossRef]

12. Liu, Z.; Geng, Z.; Hu, X. An Approach to Suppress Low Frequency Oscillation in the Traction Network of High-Speed Railway Using Passivity-Based Control. IEEE Trans. Power Syst. 2018, 33, 3909-3918. [CrossRef]

13. Liu, Z.; Geng, Z.; Wu, S.; Hu, X.; Zhang, Z. A Passivity-Based Control of Euler-Lagrange Model for Suppressing Voltage Low-Frequency Oscillation in High-Speed Railway. IEEE Trans. Ind. Inform. 2019, 15, 5551-5560. [CrossRef]

14. Ortigoza, R.S.; Juárez, J.N.A.; Sánchez, J.R.G.; Guzmán, V.M.H.; Cervantes, C.Y.S.; Taud, H. A Sensorless Passivity-Based Control for the DC/DC Buck Converter-Inverter-DC Motor System. IEEE Lat. Am. Trans. 2016, 14, 4227-4234. [CrossRef]

15. Zhang, M.; Borja, P.; Ortega, R.; Liu, Z.; Su, H. PID Passivity-Based Control of Port-Hamiltonian Systems. IEEE Trans. Autom. Control 2018, 63, 1032-1044. [CrossRef]

16. Jung, D.; Bang, S. Posture Stabilization of Wheeled Mobile Robot Based on Passivity-Based Robust Switching Control with Model Uncertainty Compensation. Appl. Sci. 2019, 9, 5233. [CrossRef]

17. Stein, G. Respect the unstable. IEEE Control Syst. Mag. 2003, 23, 12-25.

18. Yadav, S.; Verma, S.K.; Nagar, S.K. Optimized PID Controller for Magnetic Levitation System. IFAC-Pap. 2016, 49, 778-782. [CrossRef]

19. Zhai, M.; Long, Z.; Li, X. Fault-Tolerant Control of Magnetic Levitation System Based on State Observer in High Speed Maglev Train. IEEE Access 2019, 7, 31624-31633. [CrossRef]

20. Gupta, D.; Suman, S.K.; Kumar, A. Approximation Based Optimal Control Design Strategy for the Magnetic Levitation System. J. Electron. Des. Technol. 2019, 10, 8-14.

21. Sinha, P.K.; Pechev, A.N. Nonlinear H/sub /spl infin// controllers for electromagnetic suspension systems. IEEE Trans. Autom. Control 2004, 49, 563-568. [CrossRef] 